\title{
Reliability and Validity of the Japanese Version of BEMIB Modified for Patients With Bipolar Disorder: a Self-rating Scale for Medication Adherence
}

\author{
Tatsuya Tokura, M.D., ${ }^{1}$ Hiroyuki Kimura, M.D., Ph.D., ${ }^{1}$ Akira Yoshimi, Ph.D., ${ }^{1,2}$ Mitsuki \\ Ohashi, M.S., ${ }^{2}$ Mie Masuda, B.S., ${ }^{3}$ Koji Senzaki, B.S., ${ }^{3}$ Keizo Yoshida, M.D., Ph.D., ${ }^{4}$ \\ Branko Aleksic, M.D., Ph.D., ${ }^{1}$ Yukihiro Noda, Ph.D., ${ }^{2}$ Kiyofumi Yamada, Ph.D.,, \\ and Norio Ozaki, M.D., Ph.D. ${ }^{1}$ \\ ${ }^{1}$ Department of Psychiatry, Nagoya University Graduate School of Medicine, Nagoya, Japan \\ ${ }^{2}$ Division of Clinical Sciences and Neuropsychopharmacology, \\ Faculty of Pharmacy, Meijo University, Nagoya, Japan \\ ${ }^{3}$ Department of Neuropsychopharmacology and Hospital Pharmacy, \\ Nagoya University Graduate School of Medicine, Nagoya, Japan \\ ${ }^{4}$ Health Care Promotion Department, Denso Corporation, Kariya, Japan
}

\begin{abstract}
Purpose: Accurate evaluation of medication adherence is important; however, no simple evaluation scale that is applicable to patients with bipolar disorder has been established in Japan. In this study, we prepared a modified Japanese version of a self-rating scale for medication adherence in the field of psychiatry, the Brief Evaluation of Medication Influences and Beliefs (BEMIB), and investigated its reliability and validity.

Methods: Forty-one patients with bipolar disorder who visited several facilities, including Nagoya University Hospital, from April 2006 to August 2006 and from April 2009 to July 2009 underwent medication adherence evaluations using the Japanese versions of BEMIB and the Drug Attitude Inventory-10 Questionnaire (DAI-10).

Results: The Cronbach $\alpha$ coefficient of the Japanese version of BEMIB was 0.73 . Four-week test-retest reliability coefficients of each item and the BEMIB total score were 0.39-0.68 $(\mathrm{p}<0.05)$ and the intra-class correlation coefficient was $0.63(95 \% \mathrm{CI}=0.33-0.75, \mathrm{p}<0.001)$. In addition, a significant positive correlation was observed between the BEMIB and DAI-10 total scores (Pearson's correlation coefficient $=0.39, \mathrm{p}<0.001$ ), showing that the concurrent validity was sufficient.

Discussion: The Japanese version of BEMIB modified for patients with bipolar disorder is sufficiently reliable and valid. We suggest that this simple evaluation scale of medication adherence in patients with bipolar disorder is applicable in routine medical practice.
\end{abstract}

\section{Keywords: BEMIB, modified Japanese version, medication adherence, reliability, validity}

Received July 11, 2012 / Accepted November 13, 2012 / Published December 21, 2012

\section{INTRODUCTION}

Adherence requires a positive attitude on the part of the patients - accepting one's own disease and actively participating in deciding on a therapeutic policy. In a study in which physicians evaluated the adherence of patients by disease, adherence was worse in patients with chronic diseases, such as hypertension, diabetes and schizophrenia, than in people with headache or acute infections [1]. Specifically, adherence in mental disorders was lower than in patients with other chronic diseases. In psychiatric patients, medication adherence

Corresponding Author: Hiroyuki Kimura, M.D., Ph.D., Department of Psychiatry, Nagoya University Graduate School of Medicine, 65 Tsurumai, Showa, Nagoya, Aichi 466-8550, Japan Tel.: +81527442282Ｆax:+81527442293Ｅ-mail: kimurahi@med.nagoya-u.ac.jp 
was associated with prognosis, indicating the importance of adherence for treatment success [2].

Medication adherence in the field of psychiatry has been investigated in many studies, including studies in schizophrenic patients [3,4]. In addition, the importance of medication adherence in improving prognosis in bipolar disorder has been attracting an increasing amount of attention [5,6]. Specifically, it has been reported that medication adherence in bipolar disorder patients is low (54-66\%) [7-9], and that the recurrence rate is increased [10] and the incidence of suicidal behavior is increased five-fold [11] when medication adherence is poor.

To improve medication adherence, it first needs to be accurately evaluated. There are various evaluation methods, such as pill counting, measurement of the blood drug concentration, interview-based evaluations by physicians and self-rating scales. All of these methods have advantages and disadvantages [12]. Pill counting lacks reliability because the ingestion of drugs cannot be confirmed [13]. Measurement of the drug concentration in blood is a direct and objective method, but it has disadvantages, such as interindividual pharmacokinetic variability, interactions with food or other drugs, and favorable findings resulting from the re-initiation of medication for only a few days. Moreover, only a few drugs can be readily measured at clinical sites. Physician use of a structured interview that includes an evaluation scale, the Rating of Medication Influence (ROMI), has been reported [14]; however, this rating instrument is not used in routine clinical practice because of the training required for the person performing the interview, and because of the total amount of time needed to complete the interview. In a study in which the adherence of schizophrenic outpatients was evaluated by various methods, the proportion of patients considered nonadherent was 3\% in self-reports, but $24 \%$ and $25 \%$ in physician evaluation and pill counting, respectively, and $52 \%$ in an evaluation method in which an electronic device, the Electronic Medication Monitor (EMM), was used. These results clearly show that there was extreme inconsistency among the methods [15].

Of the adherence evaluation methods, self-rating scales are simple and have been shown to be useful, although overestimation and recall bias are likely to occur in self-evaluation [11]. In a study mostly in bipolar disorder and schizophrenia, the adherence level estimated from the blood drug level was significantly correlated with the results obtained using self-evaluation [16]. In another study, the consistency with objective data (e.g., pill counting, plasma drug concentration, electronic measures) was higher than that of interview-based evaluation performed by physicians [17]. Although self-rating scales are useful, as described above, there are few instruments that have been translated into Japanese. Typical evaluation scales generally used in Western countries include the Brief Evaluation of Medication Influences and Beliefs (BEMIB) [18] and the Drug Attitude Inventory-10 Questionnaire (DAI-10) [19]. A Japanese version of DAI-10 [20] exists, and its application and rating system are simple; however, it also has the disadvantage that medication behavior is not evaluated. In other words, DAI-10 may evaluate compliance rather than adherence. BEMIB is another useful self-rating scale, prepared by Dolder and coauthors in 2004 (see reference 18, Fig. 1); its reliability and validity have been demonstrated in a study in sixty-three psychiatric outpatients in which adherence was investigated for six months based on refills. BEMIB is shorter than DAI-10, avoids the above disadvantages of DAI-10 and does not require training; however, a Japanese version is not yet available.

In the present study, we prepared a modified Japanese version of BEMIB, with the aim of establishing a self-rating scale in Japanese for the simple evaluation of medication adherence in patients with bipolar disorder, in whom medication adherence markedly influences the prognosis. We also investigated the reliability and validity of this scale.

\section{SUBJECTS AND METHODS}

\section{Subjects}

The subjects were 47 patients who were treated as outpatients at Nagoya University Hospital or its affiliated sites, Yagoto Hospital and Hinaga General Center for Mental Health, from April 2006 to August 2006 and from April 2009 to July 2009. The patients had received diagnoses of bipolar disorder type I or II using the bipolar disorder section of the Structured Clinical Interview for DSM-IV Axis I disorders (SCID-I) [21], administered by expert psychiatrists. Patients with concurrent Axis I or II disorders (e.g., anxiety disorder, dementia and personality disorder) were excluded. Written informed consent was obtained from each participant after a full explanation of the study. This study was approved by the Ethics Committee of Nagoya University. 


\section{Methods}

\section{Preparation of Japanese version of BEMIB}

After obtaining the consent of the author, the original BEMIB was carefully translated into Japanese by Japanese researchers including a bilingual psychiatrist whose first language is English. In order to apply BEMIB to patients with bipolar disorder, we translated the term "antipsychotic medication" to "psychotropic medication" in Japanese, with the permission of the original author. BEMIB is based on the modified health belief model [22], and its questions encompass all domains considered to influence medication adherence: benefits of treatment, risk of illness, costs of treatment, barriers to treatment and cues to act. BEMIB is composed of eight statements, each of which is evaluated using a 5-point Likert-type scale from 1 (completely disagree) to 5 (completely agree). A high score means favorable medication adherence, except for in statements 3 and 5 , for which a low score represents high-level adherence.

\section{Reliability}

Evaluation of the modified Japanese version of BEMIB was repeated within four weeks to investigate test-retest reliability. To investigate the internal consistency reliability, the Cronbach $\alpha$ coefficient was determined. To avoid performance bias, the subjects were only told that this was a "study of an evaluation scale": there was no specific mention of "medication adherence."

\section{Validity}

To investigate the concurrent validity, the patients were simultaneously evaluated with the Japanese version of DAI-10 and with the modified Japanese version of BEMIB. The Japanese versions of the Beck Depression Inventory (BDI) [23] and the Altman Self-Rating Mania Scale (ASRM) [24] were used to evaluate depressive and manic symptoms, respectively. Since the clinical condition of a patient can change, the assessments performed using the Japanese versions of BEMIB, DAI-10, BDI and ASRM were repeated after twelve weeks. Pearson's correlation coefficients between the total scores of the modified Japanese version of BEMIB and the other scales were calculated. The scores at 0 and 12 weeks were used to analyze the correlation between the scales.

\section{Data analysis}

Statistical data are expressed as mean \pm SD. Statistical analysis was performed using SPSS for Windows Version 20.0J. Significance was determined at the 0.05 level in all tests.

\section{RESULTS}

\section{Characteristics of subjects}

Of the 47 participants, one patient with major depressive disorder and five patients who could not complete one of the evaluation scales were excluded, leaving 41 patients who were ultimately included in the analysis. No patients dropped out during the study period. There were 22 males (54\%) and 19 females (46\%), and the mean age was $50.0 \pm 12.3$ years $(27-73$ years). The diagnosis was bipolar I disorder in 24 patients $(59 \%)$ and bipolar II disorder in 17 patients (41\%) (Tables 1,2).

Table 1. Patient characteristics

\begin{tabular}{lll}
\hline Diagnosis & Bipolar I Disorder & $23(57 \%)$ \\
& Bipolar II Disorder & $18(43 \%)$ \\
Gender & Male & $22(54 \%)$ \\
& Female & $19(46 \%)$ \\
Age (years) & & $50.0 \pm 12.4$ \\
Duration of illness (years) & & $16.1 \pm 13.8$ \\
Mood stabilizers & Lithium & $28(68 \%)$ \\
& Valproate & $18(44 \%)$ \\
Dose of mood stabilizers (mg/day) & Carbamazepine & $1(2 \%)$ \\
& Lithium & $596 \pm 199$ \\
& Valproate & $705 \pm 280$ \\
& Carbamazepine & $1000 \pm 0$ \\
\hline
\end{tabular}


Table 2. Detailed patient characteristics

\begin{tabular}{|c|c|c|c|c|c|c|c|}
\hline No. & Diagnosis & Gender & $\begin{array}{l}\text { Age } \\
(y)\end{array}$ & $\begin{array}{l}\text { Duration } \\
\text { of illness } \\
\text { (m) }\end{array}$ & $\begin{array}{l}\text { Mood stabilizers } \\
\text { (mg/day) }\end{array}$ & $\begin{array}{c}\text { Antipsychotics } \\
\text { (mg/day) }\end{array}$ & Occupational Status \\
\hline 1 & BP-I & $\mathrm{m}$ & 32 & 14 & Li 800 & & white-collar worker \\
\hline 2 & BP-II & $\mathrm{m}$ & 59 & 200 & VPA 600 & & white-collar worker \\
\hline 3 & BP-II & $\mathrm{f}$ & 45 & 188 & Li 400 & & housewife \\
\hline 4 & BP-II & $\mathrm{m}$ & 36 & 57 & Li 600 & & blue-collar worker \\
\hline 5 & BP-I & $\mathrm{m}$ & 58 & 68 & Li 800 & & retired \\
\hline 6 & BP-II & $\mathrm{f}$ & 42 & 75 & VPA 600 & & housewife \\
\hline 7 & BP-I & $\mathrm{f}$ & 65 & 600 & Li 400, VPA 400 & & housewife \\
\hline 8 & BP-I & $\mathrm{f}$ & 33 & 63 & Li 600 & & white-collar worker \\
\hline 9 & BP-I & $\mathrm{f}$ & 59 & 79 & Li 400 & APZ 6 & housewife \\
\hline 10 & BP-I & $\mathrm{m}$ & 51 & 21 & Li 500 & & white-collar worker \\
\hline 11 & BP-II & $\mathrm{f}$ & 62 & 313 & Li 400 & & housewife \\
\hline 12 & BP-I & $\mathrm{m}$ & 65 & 444 & Li 400 & & white-collar worker \\
\hline 13 & BP-I & $\mathrm{f}$ & 37 & 210 & Li 300, VPA 100 & RIS 2 & salesperson \\
\hline 14 & BP-I & $\mathrm{f}$ & 73 & 308 & Li 400, VPA 600 & & housewife \\
\hline 15 & BP-I & $\mathrm{f}$ & 57 & 358 & Li 600 & RIS 1 & housewife \\
\hline 16 & BP-II & $\mathrm{m}$ & 64 & 39 & Li 300, VPA 600 & & farmer \\
\hline 17 & BP-II & $\mathrm{f}$ & 42 & 100 & VPA 800 & & housewife \\
\hline 18 & BP-II & $\mathrm{f}$ & 48 & 220 & Li 800 & & housewife \\
\hline 19 & BP-I & $\mathrm{m}$ & 42 & 136 & Li 1000 & QTP 400 & retired \\
\hline 20 & BP-I & $\mathrm{m}$ & 61 & 103 & Li 600 & RIS 1 & retired \\
\hline 21 & BP-II & $\mathrm{f}$ & 38 & 80 & VPA 600 & & housewife \\
\hline 22 & BP-II & $\mathrm{f}$ & 71 & 43 & VPA 400 & QTP 50 & housewife \\
\hline 23 & BP-I & $\mathrm{f}$ & 51 & 384 & Li 800, VPA 1200 & & housewife \\
\hline 24 & BP-II & $\mathrm{m}$ & 35 & 156 & VPA 600 & & care worker \\
\hline 25 & BP-I & $\mathrm{m}$ & 29 & 87 & Li 600, VPA 1000 & & rehabilitation helper \\
\hline 26 & BP-I & $\mathrm{m}$ & 36 & 28 & VPA 1000 & & blue-collar worker \\
\hline 27 & BP-I & $\mathrm{m}$ & 66 & 468 & VPA 1200 & QTP 750 & retired \\
\hline 28 & BP-I & $\mathrm{m}$ & 37 & 21 & VPA 600 & & blue-collar worker \\
\hline 29 & BP-II & $\mathrm{m}$ & 55 & 444 & $\mathrm{Li} 400$ & & blue-collar worker \\
\hline 30 & BP-II & $\mathrm{m}$ & 48 & 72 & VPA 800 & & white-collar worker \\
\hline 31 & BP-I & $\mathrm{f}$ & 69 & 591 & $\mathrm{Li} 400$ & & retired \\
\hline 32 & BP-I & $\mathrm{m}$ & 54 & 312 & Li 800 & QTP 500 & blue-collar worker \\
\hline 33 & BP-I & $\mathrm{f}$ & 58 & 366 & Li 600 & & housewife \\
\hline 34 & BP-I & $\mathrm{m}$ & 45 & 197 & CBZ 1000 & & unemployed \\
\hline 35 & BP-II & $\mathrm{f}$ & 27 & 43 & VPA 1000 & QTP 200 & blue-collar worker \\
\hline 36 & BP-II & $\mathrm{m}$ & 43 & 147 & Li 1000 & QTP 200 & white-collar worker \\
\hline 37 & BP-II & $\mathrm{m}$ & 53 & 75 & Li 800 & QTP 50 & teacher \\
\hline 38 & BP-I & $\mathrm{f}$ & 51 & 67 & VPA 600 & & housewife \\
\hline 39 & BP-I & $\mathrm{m}$ & 52 & 192 & Li 800 & OLZ 20 & unemployed \\
\hline 40 & BP-I & $\mathrm{f}$ & 63 & 488 & Li 600 & RIS 2 & housewife \\
\hline 41 & BP-I & $\mathrm{m}$ & 39 & 84 & Li 600 & QTP 200 & unemployed \\
\hline
\end{tabular}

BP: Bipolar Disorder Li: Lithium VPA: Valproic Acid CBZ: Carbamazepine APZ: Aripiprazole RIS: Risperidone QTP: Quetiapine

\section{Results for each evaluation scale}

A negative correlation was confirmed between the scores of statements 3 and 5 of the modified Japanese version of BEMIB and the total scores of the Japanese version of DAI-10. In contrast, we detected a positive correlation between the scores of all other statements of the modified Japanese version of BEMIB and the total scores of the Japanese version of DAI-10. As with the original version of BEMIB, it was considered adequate to invert the scores of two statements.

The mean total score of the modified Japanese version of BEMIB was $28.7 \pm 4.6$, and the mean total score of the Japanese version of DAI-10 was $4.89 \pm 4.03$.

The mean total scores of the modified Japanese ver- 
sion of BDI on the first and second evaluations were $15.3 \pm 12.1$ and $14.0 \pm 12.5$, respectively, showing no significant difference between the two evaluations by paired t-test $(\mathrm{p}=0.47)$. The mean total scores of the Japanese version of ASRM were 4.36 \pm 3.26 and 3.95 \pm 2.91 , again showing no significant difference between the two evaluations by paired t-test $(\mathrm{p}=$ $0.48)$.

\section{Reliability}

The four-week test-retest reliability coefficients of each item and total score of BEMIB ranged from 0.39 to $0.68(\mathrm{p}<0.05$, Table 3$)$ and the intra-class correlation coefficient (ANOVA-ICC) was 0.63 (95\% confidence interval $[\mathrm{CI}]=0.33-0.75, \mathrm{p}<0.001)$. The Cronbach $\alpha$ coefficient was 0.73 . In the analysis of test-retest reliability, we surveyed the medical records of all the patients and confirmed that their moods were stable and they did not experience any mood episodes, and that they were treated on an outpatient basis during the four weeks.

\section{Validity}

Regarding the concurrent validity, the correlation level was determined by calculating Pearson's correlation coefficient. The total score of the modified Japanese version of BEMIB correlated significantly with the DAI-10 total score (correlation coefficient: $0.39 ; \mathrm{p}<$ 0.001). However, no significant correlation was observed between the total scores of the modified Japanese version of BEMIB and BDI (correlation coefficient: $-0.14 ; p=0.24$ ). In addition, there was no significant correlation between the total score of the modified Japanese version of BEMIB and the ASRM total score (correlation coefficient: $0.01, p=0.91$ ).

Table 3. Four-week test-retest reliability coefficients

for the modified Japanese version of BEMIB

\begin{tabular}{ccc}
\hline Item & $\mathrm{r}$ & $\mathrm{p}$ value \\
1 & 0.42 & 0.006 \\
2 & 0.53 & 0.000 \\
3 & 0.64 & 0.000 \\
4 & 0.57 & 0.000 \\
5 & 0.61 & 0.000 \\
6 & 0.39 & 0.012 \\
7 & 0.45 & 0.003 \\
8 & 0.68 & 0.000 \\
TOTAL & 0.58 & 0.000 \\
\hline$r$ Pearson's correlation coefficient \\
\hline
\end{tabular}

\section{DISCUSSION}

Our study suggested that the modified Japanese version of BEMIB is sufficiently reliable. Using a four-week test-retest method, the score for each item and the total score of the modified Japanese version of BEMIB were significantly correlated; the intra-class correlation coefficient (ANOVA-ICC) was 0.63. According to a report describing the criteria of ANOVA-ICC in psychiatric clinical research [25], a value of 0.6 or higher is "satisfactory," suggesting that the Japanese version of BEMIB has satisfactory test-retest reliability. Moreover, the Cronbach $\alpha$ coefficient (0.73) was also acceptable, showing sufficient internal reliability.

According to our data, the modified Japanese version of BEMIB has satisfactory internal reliability and validity. Regarding the concurrent validity, the total scores of DAI-10 and the original BEMIB were significantly correlated (correlation coefficient: $0.55, p$
$<0.001$ ) [18]. A significant positive correlation was also noted between the total scores of the Japanese version of DAI-10 and the modified Japanese version of BEMIB, suggesting that the concurrent validity was also sufficient. However, the correlation coefficient shown in our study is not as high as that shown by the original BEMIB. The DAI-10 lacks evaluation of medication behavior, and the Japanese version of BEMIB used in this study was modified for patients with bipolar disorder; these factors may account for the difference between the correlation coefficient shown in our study and that for the original BEMIB.

Only a few studies have focused on the association between changes in mood and medication adherence in patients with bipolar disorder [26]. The modified Japanese version of BEMIB was not significantly correlated with the Japanese version of BDI or ASRM. Depressive symptoms and manic symptoms may occur in patients with bipolar disorder, even though their medication adherence is good. If good adherence 
becomes a habit, mood symptoms may not directly compromise adherence. Therefore, the scores of BDI and ASRM could change even when the BEMIB score remains stable. Moreover, higher adherence may lead to lower correlation between the scores of the modified Japanese version of BEMIB and BDI or ASRM. Actually, many previous studies have described poor insight as a factor influencing medication adherence, rather than symptom severity [27-29].

The modified Japanese version of BEMIB is advantageous in several respects compared with other scales designed for the evaluation of medication adherence. First, BEMIB does not require training and can be readily carried out in routine medical practice. Increases in total score indicate improved adherence. It is possible for patients to complete the scale by themselves while waiting for outpatient consultation; in our study, all patients could complete the scale within three minutes. Second, it can be combined with an education plan. Factors reducing medication adherence can be identified, facilitating investigation of a psychoeducational approach to improving adherence. It may also be possible to investigate changes in medication adherence caused by therapeutic interventions. Third, the modified Japanese version of BEMIB overcomes the problems of one of the few self-rating scales available in the Japanese language, DAI- 10. The validity of DAI-10 is based on judgments made by physicians, and is disadvantageous in that medication behavior is not evaluated, but the modified Japanese version of BEMIB resolves this issue. For example, statement 4 of the modified Japanese version of BEMIB is: "I have a system (e.g., pill box, medication calendar, someone giving me my medication) that helps me remember to take my psychotropic medication." Finally, the statements are applicable to various treatment methods, and the scale is not limited only to pharmacological therapy.

Several limitations must be considered when interpreting the study findings. First, the sample size was relatively small. The sample size of the article describing the original study focused on BEMIB was also relatively small, with only 63 patients. As our study had only 41 patients, the statistical power may have been reduced, and consequently the possibility of a type 2 error cannot be completely ruled out. Second, since we investigated the reliability and validity only for bipolar disorder in this study, it was impossible to determine if the same results could be obtained in other psychiatric disorders. However, the statements in the modified Japanese version of BEMIB are not limited to bipolar disorder, and could potentially be applied to other psychiatric disorders. In this study, the original BEMIB was modified in translation from English to Japanese in order to evaluate medication adherence in patients with bipolar disorder. Back translation was not performed because the original BEMIB and its modified Japanese version are not identical in content. This means that the BEMIB modified for patients with bipolar disorder is available only in Japanese, not in English.

This study showed the usefulness of the modified Japanese version of BEMIB as an evaluation scale of medication adherence in the field of psychiatry using a sample that consisted of patients suffering from bipolar disorder.

\section{CONCLUSIONS}

This study demonstrated the sufficient reliability and validity of the modified Japanese version of BEMIB for patients with bipolar disorder. This is a useful tool well suited for the evaluation of medication adherence in patients with bipolar disorder, without training in routine medical practice.

\section{ACKNOWLEDGEMENTS}

We sincerely thank the patients for their cooperation in this study.

\section{CONFLICTS OF INTEREST}

All authors declare that they have no conflicts of interest.

\section{REFERENCES}

[1] Keith SJ, Kane JM. Partial compliance and patient consequences in schizophrenia: our patients can do better. J Clin Psychiatry 2003; 64: 1308-1315.

[2] Osterberg L, Blaschke T. Adherence to medication. N Engl J Med 2005; 353: 487-497.

[3] Lacro JP, Dunn LB, Dolder CR, Leckband SG, Jeste DV. Prevalence of and risk factors for medication nonadherence in patients with schizophrenia: a comprehensive review of recent literature. J Clin Psychiatry 2002; 63: 892-909.

[4] Valenstein M, Blow FC, Copeland LA, et al. Poor antipsychotic adherence among patients with schizophrenia: medication and patients factors. Schizophr Bull 2004; 30: 255-264.

[5] Vieta E. Improving treatment adherence in 
bipolar disorder through psychoeducation. J Clin Psychiatry 2005; 66(Suppl 1): 24-29.

[6] Colom F, Vieta E, Tacchi MJ, Sa(a)nchezMoreno J, Scott J. Identifying and improving non-adherence in bipolar disorder. Bipolar Disord 2005; 7(Suppl 5): 24-31.

[7] Suppes T, Baldessarini RJ, Faedda GL, Tohen $\mathrm{M}$. Risk of recurrence following discontinuation of lithium treatment in bipolar disorder. Arch Gen Psychiatry 1991; 48: 1082-1988.

[8] Colom F, Vieta E, Martinez-Aran A, Reinares M, Benabarre A, Gasto C. Clinical factors associated to treatment non-compliance in euthymic bipolar patients. J Clin Psychiatry 2000; 61: 549-555.

[9] Sajatovic M, Bauer MS, Kilbourne AM, Vertrees JE, Williford W. Self-reported medication treatment adherence among veterans with bipolar disorder. Psychiatr Serv 2006; 57: 56-62.

[10] Gonzales-Pinto A, Mosquera F, Alonso M, et al. Suicidal risk in bipolar I disorder patients and adherence to long-term lithium treatment. Bipolar Disord 2006; 8: 618-624.

[11] Baldessarini RJ, Perry R, Pike J. Factors associated with treatment nonadherence among US bipolar disorder patients. Hum Psychopharmacol 2008; 23: 95-105.

[12] Basra RK, Wagg A, Chapple C, et al. A review of adherence to drug therapy in patients with overactive bladder. BJU Int 2008; 102: 774-779.

[13] Sajatovic M, Chen PJ, Dines P, et al. Psychoeducational approaches to medication adherence in patients with bipolar disorder. Dis Manag Health Out 2007; 15: 181-192.

[14] Weiden P, Rapkin B, Mott T, et al. Rating of medication influence (ROMI) scale in schizophrenia. Schizophr Bull 1994; 20: 297-310.

[15] Remington G, Kwon J, Collins A, Laporte D, Mann S, Christensen B. The use of electronic monitoring (MEMS) to evaluate antipsychotic compliance in outpatients with schizophrenia. Schizophr Res 2007; 90: 229-237.

[16] Jonsdottir H, Opjordsmoen S, Birkenaes AB, et al. Medication adherence in outpatients with severe mental disorders: relation between selfreports and serum level. J Clin Psychopharmacol 2010; 30: 169-175.

[17] Garber MC, Nau DP, Erickson SR, Aikens JE, Lawrence JB. The concordance of self-report with other measures of medication adherence. A summary of the literature. Med Care 2004; 42: 649-652.

[18] Dolder CR, Lacro JP, Warren KA, Golshan S,
Perkins DO, Jeste DV. Brief evaluation of medication influences and beliefs: development and testing of a brief scale for medication adherence. J Clin Psychopharmacol 2004; 24 : 404-409.

[19] Hogan TP, Awad AG, Eastwood R. A self-report scale predictive of drug compliance in schizophrenics: reliability and discriminative validity. Psychol Med 1983; 13: 177-183.

[20] Miyata R, Fujii Y, Inagaki A, Yagi G: Psychopharmacology for the patients with schizophrenia and their quality of life (QOL): Assessment using the Japanese version of Drug Attitude Inventory (DAI). Seishin Sinkeigaku Zasshi. 1996; 98: 1045-1046 (abstract) (in Japanese).

[21] First M, Spitzer RL, Gibbon M, William JBW. Structured Clinical Interview for DSM-IV Axis I disorders (SCID-I). Biometric Research Department, New York, 1997.

[22] Perkins DO. Adherence to antipsychotic medications. J Ciln Psychiatry 1999; 60: 25-30.

[23] Beck AT, Ward CH, Mendelson M, Mock J, Erbaugh J. An Inventory for measuring depression. Arch Gen Psychiatry 1961; 4: 561-571.

[24] Altman EG, Hedeker D, Peterson JL, Davis JM. The Altman Self-Rating Mania Scale. Biol Psychiatry 1997; 42: 948-955.

[25] Kraemer HC. Coping strategies in psychiatric clinical research. J Consult Psychol 1981; 49: 309-319.

[26] Gaudiano BA, Weinstock LM, Miller IW. Improving treatment adherence in bipolar disorder: a review of current psychosocial treatment efficacy and recommendations for future treatment development. Behav Modif 2008; 32: 267-301.

[27] David A, Buchanan A, Reed A, Almeida O. The assessment of insight in psychosis. $\mathrm{Br} \mathrm{J}$ Psychiatry 1992; 161: 599-602.

[28] Amador XF, Strauss DH, Yale SA, Flaum MM, Endicott J, Gorman JM. Assessment of insight in psychosis. Am J Psychiatry 1993; 150: 873-879.

[29] Day JC, Bentall RP, Roberts C, et al. Attitudes toward antipsychotic medication: the impact of clinical variables and relationships with health professionals. Arch Gen Psychiatry 2005; 2: 717-724. 